\title{
A review of the effect of music on dental anxiety in children
}

\author{
S. L. Ainscough ${ }^{1}$ (D) L. Windsor ${ }^{2} \cdot$ J. F. Tahmassebi ${ }^{1}$
}

Received: 6 June 2018 / Accepted: 28 September 2018 / Published online: 29 October 2018

(c) The Author(s) 2018

\begin{abstract}
Aim To review the current literature on the effectiveness of using music as an intervention to reduce dental anxiety in children.

Methods At the University of Leeds, the School of Music and the School of Dentistry collaborated to conduct an online search strategy. The Cochrane Library and Medline databases were used to find the current available evidence.

Results Systematic reviews and clinical trial studies as well as cohort studies containing pertinent information on the effect of music on anxiety in the clinical setting were reviewed. The literature showed that music can have a biological and psychological impact on emotion and consequently has been used effectively as an aid to moderate anxiety in the clinical setting. With regard to paediatric dentistry, majority of studies were found to support the use of music in reducing dental anxiety in children, however several additional studies showed that music did not significantly reduce the children's dental anxiety. The studies employed a number of methods to measure dental anxiety including the Venham's Picture Test, the Venham's clinical anxiety rating scale and pulse oximetry. They also used a range of music types; some studies allowed for patient self-selection of music whereas others dictated the music the children listened to.

Conclusions There is an increasing body of evidence to support the use of music to moderate anxiety within the clinical setting in both medicine and dentistry. However, the current evidence for the effectiveness of using music to reduce dental anxiety in children is inconclusive and of limited quality.
\end{abstract}

keywords Music $\cdot$ Children $\cdot$ Anxiety

\section{Introduction}

Music's potential within healthcare settings and its broader application as a health and wellbeing intervention in the community has received much attention from researchers and practitioners in recent years. Although this is partly due to an increasing recognition that the creation, consumption and clinical application of the arts can bring considerable benefit (AHRC 2018), music has received special attention in this respect. Although music has not always been perceived to be beneficial to health (Kennaway 2012) there is a growing evidence- and practice-base that argues the case for a broad range of clinical and community applications

S. L. Ainscough

s.1.ainscough@leeds.ac.uk

1 School of Dentistry, Leeds Dental Institute, University of Leeds, Worsley Building, Clarendon Way,

Leeds LS2 9LU, UK

2 School of Music, University of Leeds, Leeds, UK for music in addition to the specificity of music therapy as a clinical practice (Bunt 2014).

Music is seen to have health and/or wellbeing benefits across a spectrum of practices from community choir participation to its use in waiting rooms and surgical settings as background music, both to directly influence mood and arousal levels and to distract from unpleasant thoughts and feelings. Engagement or even just exposure to music can improve mood, offset or mask pain and anxiety, play a role in enhancing cardiovascular fitness, and lead to greater social integration. The evidence-base for such applications varies in quality and generalisability, but has employed measures as diverse as diaries, interviews and questionnaires, adjective scales and standard instruments of psychological state, physiological measures of autonomic state and even the production of hormones such as acetylcholine and cortisol and brain imaging studies (MacDonald et al. 2012).

Dental anxiety currently has a high prevalence in children. The Children's Dental Health Survey in 2013 found that $21 \%$ of 5 -year-olds and $17 \%$ of 8 -year-olds were 
reported by their parents to be anxious about visiting the dentist. In older children, dental anxiety was found to be higher with $76 \%$ of 12 -year-olds and $64 \%$ of 15 -year-olds reporting either moderate or severe dental anxiety when visiting the dentist (HSCIC 2015).

In children, dental anxiety has been shown to lead to increased levels of carious and extracted teeth, increased episodes of dental pain and a subsequent reduction in their oral health quality of life. Poor oral health can not only have notable physical consequences for the child such as disturbed sleep, reduced oral intake and delayed growth and development, but it can also affect their concentration resulting in poor performance at school. Social interactions with peers can also be negatively affected and the child can become subject to bullying due to the appearance of their dentition (Morgan et al. 2016).

Dental anxiety in children has also been linked to the anxious child having had a previous negative dental experience; for example, children who have had a dental general anaesthetic are 2.5 times more likely to be dentally anxious as they get older (Haworth et al. 2017). It is therefore vital that any dental treatment provided for paediatric patients is conducted in the most reassuring and relaxed way possible using appropriate interventions and management strategies in order to reduce anxiety. For children, feared aspects of dental treatment are often sensory, with the most commonly feared aspects being the sight and feeling of the needle and the sight, sound and sensation of the drill (Taani et al. 2005).

The use of behaviour management techniques is important for the success of treatment; hence, the use of alternative therapies such as music can play a significant role in reducing anxiety.

\section{Music in clinical settings}

Music has been used in clinical settings within general practice (both medical and dental) and more specific settings such as surgery and particular professions allied to medicine (such as physiotherapy and speech therapy). It is useful at the outset to distinguish between the clinical practice of music therapists, who have specialist training (accredited in the UK by the British Association for Music Therapy) and the use of music by lay-practitioners who are not specifically trained, although they may have varying levels of practical and/or theoretical musical training, or some background in music psychology or clinical psychology.

Leaving aside the former, which is beyond the scope of this article, music has been used across the whole gamut of medicine and health, from emergency medicine (for example to reduce the need for pharmaceutical interventions) through cancer treatment and palliative care, in both physical and mental rehabilitation, and in psychiatry. Music can improve low mood, serve as a stimulus for talking therapy, moderate pain responses, and improve autonomic tone (Fancourt 2017). Most relevant here is a growing body of empirical work within surgery, both dental and medical, where music is administered either as background or directly to a patient through earphones during procedures. There is now a body of evidence that suggests that music is of benefit in the moderation of pain, stress and anxiety, and may have financial as well as clinical benefits as opposed to more traditional interventions (Spintge 2012).

\section{Music and the psychobiology of anxiety}

Music's impact on our physiological and cognitive state is far from straightforward. Reactions to music are highly individual and context dependent, varying with personality, experience, surroundings, age, taste and cultural background. Some generalities apply however, and certain patterns of musical structure (and associated lyrical content) may have predictable impacts on listeners, and there are conventional as well as more individual associations between styles and individual pieces of music, so it may be possible to predict and therefore prescribe music as a psychobiological treatment. Nonetheless, such predictable responses are elusive: for example, a piece of music might have a generally relaxing effect on most patients (due to its intrinsic musical structures), but the opposite effect on one patient who had first heard it in a shopping mall during an armed robbery (Sloboda and Juslin 2011).

Anxiety is both a physiological and psychological construct, with the two aspects influencing one another. The physiological component of anxiety is heightened arousal, which can be measured objectively either through monitoring the presence of hormones in the body, or through the impact of these and the action of the central and peripheral nervous systems on heartrate and heartrate variability, respiration and galvanic skin response, for example. Most theorists regard the heightened arousal of anxiety as the consequence of a variety of cognitive interpretations of threat or incongruity; but such cognitions also explain the interpretation of that arousal as anxiety, rather than, for example, excitement (Spielberger 2013). It is helpful in this context to visualise emotional responses to the external environment along two dimensions, one related to valence (the cognitive interpretation of the emotion from positive to negative) and the other its level of activity (high or low arousal) (Russell 1980). Anxiety sits at the extreme negative on the valence dimension, with high arousal, whereas excitement would have similar levels of arousal, but positive valence. Music has been shown to influence both biological and psychological components of emotion along these dimensions in somewhat predictable ways (Sloboda and Juslin 2011), and it 
is for this reason that one might choose to use it in a clinical setting to moderate anxiety, whether situational or chronic, above and beyond its implicit appeal.

\section{Clinical application of music in the treatment and management of anxiety}

Spintge in his review of music in operating theatres focuses extensively on the use of music to moderate stress and anxiety. He coined the term Anxioalgolytic Music (AAM) to refer to this practice, and given the individual differences of response, suggests self-chosen music as the most effective way of defining the musical material listened to, and notes that using headphones (where possible) reduces the impact of the music on staff (MacDonald et al. 2012). This does make staff-patient communication more difficult, although in noisy settings may help mask irritating or anxiety-inducing environmental sounds for the patient. Music played over loudspeakers has both positive and negative effects on operating room staff: it can disrupt effective communication, but can also produce a calm working environment (George et al. 2011; Weldon et al. 2015). What is clear is that music is being used in perioperative settings, and despite the practical issues that arise, may be an effective intervention alongside more traditional approaches to chronic or situational anxiety when undergoing procedures.

\section{Music and dental anxiety in children}

There has been limited research into whether music can be used as an effective tool to reduce anxiety in children undergoing dental procedures and currently the evidence remains inconclusive. It is believed that music may be used alongside other behaviour management techniques to reduce dental anxiety in children and its use should be considered by all.

Several studies have examined the positive effects of listening to music on anxiety and pain in dental patients. In the majority of these studies, dental patients were asked to listen to pre-recorded music through a set of headphones for the duration of the dental procedure. Marwah et al. (2005) conducted a randomised controlled trial; this study included 40 children aged between 4 and 8 years old who were divided into three groups: control group (no music), instrumental music group and nursery rhymes group. Each child had a screening visit, an oral prophylaxis visit, a restorative visit and then an extraction visit. The children's anxiety was measured using the Venham's picture test (VPT), the Venham's clinical anxiety rating scale (VCRS), pulse rate and oxygen saturation. The results showed that the VPT scores were not significantly different between the three groups and the pulse rate was not significantly different between the control group and the two music groups. However, the VCRS scores and pulse rate in the instrumental group were significantly less than in the nursery rhyme group. Overall the study found that instrumental music was more effective at reducing dental anxiety than nursery rhymes.

In a more recent randomised controlled trial by Jindal and Kaur (2011), 30 children aged between 4 and 8 years old were randomly divided into two groups, a control and a music group (self-selected music). Each child had four visits which increased in their invasiveness. The child's anxiety levels were measured using the VPT before and immediately after each appointment. The levels of anxiety were found to be greatest in the third and fourth visits as the invasiveness of the procedures increased. Interestingly, the reported anxiety levels were significantly reduced in the music group.

Similarly, Singh et al. (2014) in a randomised controlled trial found that listening to patient selected music significantly decreased intra-operative anxiety compared with the no music control group in 60 children aged between 6 and 12 years old undergoing dental extractions. Physiological anxiety measurements were recorded using a pulse oximeter and blood pressure monitor. In addition, self-reported anxiety measurement using the VPT was also used. The study showed that systolic blood pressure, pulse rate and VPT scores were all significantly reduced in the music group.

In contrast to the above studies, several others have shown that music is not an effective tool to reduce dental anxiety in paediatric patients. Aitken et al. (2002) concluded that music did not result in a reduction in pain, anxiety or uncooperative behaviour during dental procedures in children aged between 4 and 6 years old. The children were not given a choice of music to listen to which the authors' recognised could have prevented the child from fully benefiting from the music as it was not familiar to them; however $93 \%$ of the patients who listened to the music said that they enjoyed listening to it. In addition, the patients included in the study were relatively young at 4-6 years old compared with other studies. It may be that older children are more likely to benefit from the effects of music.

In another study by Gupta et al. (2017), 60 patients aged between 3 and 7 years old who required treatment under local analgesia were divided into three groups: a control group, an upbeat music group and a relaxing music group. In the first visit all the children had restorative treatment following an inferior alveolar nerve block in one quadrant. At the following visit approximately 2 weeks later, the children wore headphones; the music groups listened to music and for the control group no music was played. For both visits, anxiety was measured using the VPT pre and post-operatively, a 
visual analogue scale post-operatively to assess the child's perceived pain and heart rate was measured using a pulse oximeter before treatment, during local analgesia and at subsequent 5 min intervals. The child was also filmed and their behaviour assessed using the North Carolina Behaviour Rating Scale. The study concluded that music did not significantly reduce pain, anxiety or disruptive behaviour.

While the use of music has been explored within various clinical settings in both medicine and dentistry, the evidence-base for its use in reducing dental anxiety in children remains inconclusive and of limited quality. In most of the studies included in this article, there was limited discussion with regard to ethical approval, how the participants were recruited, how the participants were placed into different treatment groups and where they stated that they were 'randomly allocated', they did not mention the method of randomisation. There was also little reference as to whether the clinicians were blinded to the treatment group and any potential sources of bias.

\section{Conclusion}

Music has been shown to influence both the biological and psychological aspects of anxiety and there is a growing body of evidence to support its use alongside or instead of traditional interventions within the clinical setting.

Open Access This article is distributed under the terms of the Creative Commons Attribution 4.0 International License (http://creativeco mmons.org/licenses/by/4.0/), which permits unrestricted use, distribution, and reproduction in any medium, provided you give appropriate credit to the original author(s) and the source, provide a link to the Creative Commons license, and indicate if changes were made.

\section{References}

Aitken JC, Wilson S, Coury D, Moursi AM. The effect of music distraction on pain, anxiety and behavior in pediatric dental patients. Paediatr Dent. 2002;24:114-8.

Arts and Humanities Research Council. https://ahrc.ukri.org/newse vents/news/all-party-parliamentary-inquiry-into-arts-health-andwellbeing-launches-report/. Accessed 18 May 2018.

Bunt L, Stige B. Music therapy and art beyond words. Oxford: Routledge; 2014.
Cohen SM, Fiske J, Newton JT. Behavioural dentistry: the impact of dental anxiety on daily living. Br Dent J. 2000;189:385-90.

Fancourt D. Arts in health: designing and researching interventions. Oxford: Oxford University Press; 2017.

George S, Ahmed S, Mammen KJ, John GM. Influence of music on operation theatre staff. J Anaesthesiol Clin Pharmacol. 2011;27(3):354-7.

Gupta N, Gupta H, Gupta P, Gupta N. Evaluation of the role of music as a nonpharmacological technique in management of child patients. J Contemp Dent Pract. 2017;18(3):194-7.

Haworth S, Dudding T, Waylen A, Thomas SJ, Timpson NJ. Ten years on: is dental general anaesthesia in childhood a risk factor for caries and anxiety? Br Dent J. 2017;222:299-304.

Health and Social Care Information Centre. Children's dental health survey 2013 report 1: attitudes, behaviours and children's dental health: Health and Social Care Information Centre; 2015. http:// www.hscic.gov.uk/catalogue/PUB 17137/CDHS2013-Repor t1-Attitudes-and-Behaviours.pdf. Accessed 23 May 2018.

Jindal R, Kaur R. Can we tune our pediatric patients? Int J Clin Pediatr Dent. 2011;4(3):186-9.

Kennaway J. Bad vibrations: the history of the idea of music as a cause of disease. Oxford: Routledge; 2012.

Klingberg G, Broberg AG. Dental fear/anxiety and dental behavior management problems in children and adolescents: a review of prevalence and concomitant psychological factors. Int J Pediatr Dent. 2007;17:391-406.

Locker D, Liddell A, Dempster L, Shapiro D. Age of onset of dental anxiety. J Dent Res. 1999;78(3):790-6.

MacDonald R, Kreutz G, Mitchell L, Music. Health and wellbeing. Oxford: Oxford University Press; 2012.

Marwah N, Probhakar AR, Raja OS. Music distraction-its efficacy in management of anxious pediatric dental patients. J Indian Soc Pedodontics Prev Dent 2005;23(4):168-170.

Morgan AG, Rodd H, Porritt JM, et al. Children's experiences of dental anxiety. Int J Pediatr Dent. 2016;27(2):87-97.

Russell JA. A circumplex model of affect. J Pers Soc Psychol. 1980;39(6):1161-78.

Singh D, Samadi F, Jaiswal JN, Tripathi AN. Stress reduction through audio distraction in anxious pediatric dental patients: an adjunctive clinical study. Int J Clin Paediatr Dent. 2014;7(3):149-52.

Sloboda JA, Juslin PN. Handbook of music and emotion: theory, research, applications. Oxford: Oxford University Press; 2011.

Spintge R. Clinical use of music in operating theatres. In: MacDonald R, Kreutz G, Mitchell L, editors. Music health and wellbeing. Oxford: Oxford university press; 2012. pp. 266-286.

Spielberger CD. Anxiety. Current trends in theory and research. New York: Elsevier; 2013.

Taani DQ, El-Qaderi SS, Abu Alhaija ESJ. Dental anxiety in children and its relationship to dental caries and gingival condition. Int $\mathbf{J}$ Dent Hyg. 2005;3(2):83-7.

Weldon SM, Korkiakangas T, Bezemer J, Kneebone R. Music and communication in the operating theatre. J Adv Nurs. 2015;71(12):2763-74. 